\title{
Low-temperature Synthesis of $\mathrm{FeTe}_{0.5} \mathrm{Se}_{0.5}$ Polycrystals with a High Transport Critical Current Density
}

\author{
Qing-Ping Ding ${ }^{1,2}$, Shyam Mohan ${ }^{1}$, Yuji Tsuchiya ${ }^{1}$, Toshihiro Taen ${ }^{1}$, \\ Yasuyuki Nakajima ${ }^{1,2}$ and Tsuyoshi Tamegai ${ }^{1,2}$ \\ ${ }^{1}$ Department of Applied Physics, The University of Tokyo, 7-3-1 Hongo, Bunkyo-ku, \\ Tokyo 113-8656, Japan \\ ${ }^{2}$ JST, Transformative Research-Project on Iron Pnictides (TRIP), 7-3-1 Hongo, \\ Bunkyo-ku, Tokyo 113-8656, Japan
}

PACS: 74.70.Xa, 74.25.F-, 74.25.Ha, 74.25.Sv, 74.62.Bf

\begin{abstract}
We have prepared high-quality polycrystalline $\mathrm{FeTe}_{0.5} \mathrm{Se}_{0.5}$ at temperature as low as $550^{\circ} \mathrm{C}$. The transport critical current density evaluated by the current-voltage characteristics is over $700 \mathrm{~A} / \mathrm{cm}^{2}$ at $4.2 \mathrm{~K}$ under zero field, which is several times larger than $\mathrm{FeTe}_{0.5} \mathrm{Se}_{0.5}$ superconducting wires. The critical current density estimated from magneto-optical images of flux penetration is also similar to this value. The upper critical field of the polycrystalline $\mathrm{FeTe}_{0.5} \mathrm{Se}_{0.5}$ at $T=0 \mathrm{~K}$ estimated by Werthamer-Helfand-Hohenberg theory is $585 \mathrm{kOe}$, which is comparable to that of single crystals. This study gives some insight into how to improve the performance of $\mathrm{FeTe}_{0.5} \mathrm{Se}_{0.5}$ superconducting wires.
\end{abstract}

Motivated by the discovery of superconductivity in oxypnictide $\mathrm{LaFeAsO}_{1-\mathrm{x}} \mathrm{F}_{\mathrm{x}}$ with a critical temperature $T_{\mathrm{c}} \sim 26 \mathrm{~K}$, a series of iron-based superconductors have been synthesized in a short time [1-8]. Among these compounds, $\mathrm{FeSe}$ and $\mathrm{FeTe}_{1-\mathrm{x}} \mathrm{Se}_{\mathrm{x}}(11$ system) attracted much attention because of its simpler planar crystal structure than layered iron-pnictides. Therefore, they are better candidates to investigate the underlying mechanism of superconductivity in the iron-based system. The lower toxicity and less sensitivity to atmosphere of starting materials also make 11 system more promising compared with iron-pnictide superconductors. Although the transition temperature, $T_{\mathrm{c}}$, is not very high in this 11 system, it can be enhanced to as high as $37 \mathrm{~K}$ by applying pressure $[9,10]$. Recently, by introducing alkali metal $\mathrm{K}(\mathrm{Cs}, \mathrm{Rb})$ into this 11 system to form a ternary compound, $T_{\mathrm{c}}$ is increased up to $30 \mathrm{~K}$ [11-13]. In addition to the very simple crystal structure, the very high values of upper critical field make this system a potential candidate for practical applications [14-18]. Also critical current density, $J_{\mathrm{c}}$, in a high-quality single crystal is reported to be over $1 \times 10^{5} \mathrm{~A} / \mathrm{cm}^{2}$ [18-20]. Superconducting wires made from $\mathrm{FeTe}_{0.5} \mathrm{Se}_{0.5}$ have been reported, but the transport critical current density is restricted to $\sim 230 \mathrm{~A} / \mathrm{cm}^{2}$ at $4.2 \mathrm{~K}$ even under zero field [21-23]. Polycrystalline samples of iron-based superconductors in general have the problem of weak links. In order to improve the performance of $\mathrm{FeTe}_{1-\mathrm{x}} \mathrm{Se}_{\mathrm{x}}$ wires, it is essential to investigate the performance of $\mathrm{FeTe}_{1-\mathrm{x}} \mathrm{Se}_{\mathrm{x}}$ polycrystals and how to reduce the weak-link features. We have reported $\mathrm{FeTe}_{1-x} \mathrm{Se}_{\mathrm{x}}$ polycrystals prepared at higher temperatures, $600^{\circ} \mathrm{C} \sim 680^{\circ} \mathrm{C}$, with less impurities [24]. Since the grain sizes prepared at higher temperatures are too large, weak links between grains are particularly obvious. Here we report the low-temperature synthesis and characterization of high quality $\mathrm{FeTe}_{1-x} \mathrm{Se}_{\mathrm{x}}$ polycrystalline sample. The characterization through X-ray diffraction, magnetization, resistivity, transport critical current density, and magneto-optical measurements are discussed.

$\mathrm{FeTe}_{0.5} \mathrm{Se}_{0.5}$ polycrystalline samples were synthesized by solid-state reaction method. Stoichiometric amounts of Fe powder (Kojundo Chemical Laboratory, 99.9\%), Te grains (same as above, 99.9999\%), and Se grains (same as above, 99.9\%) were weighed, mixed and ground with an agate mortar and pestle. The mixing and grinding processes were carried out 
in nitrogen-filled glove box. The ground powder was cold-pressed into pellets with 600 $\mathrm{kg} / \mathrm{cm}^{2}$ uniaxial pressure. The pellets were sealed in an evacuated quartz tube, and slowly ramped to $550^{\circ} \mathrm{C}$ in 5 hours from room temperature, and kept for 24 hours. After cooling to room temperature naturally, the reacted pellets were reground, pressed $\left(1800 \mathrm{~kg} / \mathrm{cm}^{2}\right.$ uniaxial pressure), sealed in a quartz tube, sintered again at $550^{\circ} \mathrm{C}$ for $24 \mathrm{~h}$, and finally annealed at $400^{\circ} \mathrm{C}$ for $24 \mathrm{~h}$, followed by quenching. When the sintering temperature is lower than $500^{\circ} \mathrm{C}$ and higher than $400^{\circ} \mathrm{C}$, although 11 phase can be formed, there are more impurities, and the superconducting volume fraction is very small.

The phase identification of the sample was carried out by means of powder X-ray diffraction (M18XHF, MAC Science) with $\mathrm{Cu}-\mathrm{K} \alpha$ radiation generated at $40 \mathrm{kV}$ and $200 \mathrm{~mA}$. Bulk magnetization is measured by a superconducting quantum interference device SQUID magnetometer (MPMS-5XL, Quantum Design). The microstructures were characterized by Scanning Electron Microscopy (SEM, Hitachi S-4300) operated at $15 \mathrm{kV}$. Resistivity and current-voltage $(I-V)$ measurements were performed by four-probe method with silver paste for contact. Resistivity measurements were performed within the sample chamber of a SQUID magnetometer. $I-V$ measurements were performed in a bath type cryostat (Spectromag, Oxford Instruments). For local magnetic characterization, we applied magneto-optical (MO) imaging. For this purpose, the sample was cut using a wire saw, and the surface was polished with a lapping film. A Bi-substituted iron-garnet indicator film is placed in direct contact with the sample, and the whole assembly is attached to the cold finger of a He-flow cryostat (Microstat-HR, Oxford Instruments) and cooled down to $5 \mathrm{~K}$. MO images are acquired by using a cooled CCD camera with 12-bit resolution (ORCA-ER, Hamamatsu). To enhance the visibility of the local magnetic induction and eliminate the signals from the impurity phases, a differential imaging technique is employed [25, 26].

Figure 1(a) shows the X-ray diffraction pattern of as-prepared polycrystalline sample. All the peaks were well indexed using a space group of $\mathrm{P} 4 / \mathrm{nmm}$ except for small peaks as an impurity phase marked by asterisks. The compound crystallizes in a tetragonal structure and the impurity phase is identified as hexagonal $\mathrm{Fe}_{7} \mathrm{Se}_{8}$. The calculated lattice constants are $a=$ $0.3799 \mathrm{~nm}$, and $c=0.5980 \mathrm{~nm}$, which are slightly smaller than the samples prepared at higher temperatures ( $a=0.3800 \mathrm{~nm}$, and $c=0.6011 \mathrm{~nm})$ and single crystals [19, 27]. Figure 1(b) is the SEM image of this sample. From this image, a typical grain size is $\sim 10 \mu \mathrm{m}$ or less although with a broad size distribution. The grains are sheet-like with the ratio of width $(w)$ to thickness $(t)$ may be larger than 10. Although the amount of impurities of this sample is higher than the sample prepared at commonly used higher temperature at $600-680^{\circ} \mathrm{C}[24]$, the average grain size has been reduced from $50 \sim 100 \mu \mathrm{m}$ to less than $10 \mu \mathrm{m}$. Temperature dependences of zero-field-cooled (ZFC) and field-cooled (FC) magnetization at 5 Oe of the $\mathrm{FeTe}_{1-\mathrm{x}} \mathrm{Se}_{\mathrm{x}}$ polycrystalline sample are shown in Fig. 1(c). The sample shows an onset of diamagnetism at around $12.5 \mathrm{~K}$. The feature of two transitions, which is due to weak links between grain boundaries and appeared in many $\mathrm{FeTe}_{1-\mathrm{x}} \mathrm{Se}_{\mathrm{x}}$ polycrystalline samples prepared at higher temperatures [22, 24], is suppressed in our present sample. So we think the reduction of grain size is beneficial to the grain boundaries.

Figure 2(a) shows temperature dependence of the resistivity of the sample. The room temperature resistivity is $690 \mu \Omega \mathrm{cm}$. The resistivity shows semiconducting behavior above $150 \mathrm{~K}$, and shows metallic behavior in the normal state below $150 \mathrm{~K}$. A sharp drop in resistivity was observed at about $14.3 \mathrm{~K}$, which indicates the onset of superconductivity. The zero resistance occurs at $12.5 \mathrm{~K}$ and the transition width is $1.8 \mathrm{~K}$. The residual resistivity ratio $\rho(300 \mathrm{~K}) / \rho\left(T_{\mathrm{c}}^{\text {onset }}\right)$ is 2.06 . Similar temperature dependence of resistivity is reported for samples with compositions close to $\mathrm{FeTe}_{0.5} \mathrm{Se}_{0.5}[19,22,27-31]$. This behavior has been attributed to weak charge-carrier localization due to a large amount of excess $\mathrm{Fe}$ in $\mathrm{Fe}_{1+y} \mathrm{Te}_{1-\mathrm{x}} \mathrm{Se}_{\mathrm{x}}$ system [27]. The value of resistivity in our polycrystalline sample is larger than Ref [27], but similar to Ref [19], and lower than that reported in Refs [22, 28, 29]. The intrinsic microscopic inhomogeneities of polycrystalline sample and impurity phase may be 
the reason for this higher value of resistivity. The variation of $T_{\mathrm{c}}$ with magnetic field is shown in figure 2 (b) for $H=0,10,20,30,40$ and $50 \mathrm{kOe}$. With increasing field, the resistive transition shifts to lower temperatures accompanied by a slight increase in the transition width. Inset of figure 2 (b) shows the variation of the upper critical field $H_{\mathrm{c} 2}$ with reduced temperature $t=T / T_{\mathrm{c}}$ onset for the $\mathrm{FeTe}_{0.5} \mathrm{Se}_{0.5}$ polycrystalline sample. The values of $H_{\mathrm{c} 2}$ were defined as the field at the midpoint of the resistive transition. The slope of $H_{\mathrm{c} 2}$ at $T_{\mathrm{c}}$ is -60.6 $\mathrm{kOe} \mathrm{K}^{-1}$. The value of $H_{\mathrm{c} 2}$ at $T=0 \mathrm{~K}$ estimated using the Werthamer-Helfand-Hohenberg formula [32], $H_{\mathrm{c} 2}(0)=-0.69 T_{\mathrm{c}}\left|\mathrm{d} H_{\mathrm{c} 2} / \mathrm{d} T\right|_{T=T \mathrm{c}}$, is $585 \mathrm{kOe}$. This value is comparable to the single crystals [14-18]. In order to extract the superconducting parameters, we have used the Ginzburg-Landau (GL) formula for the coherence length $(\xi), \xi=\left(\Phi_{0} / 2 \pi H_{\mathrm{c} 2}\right)^{1 / 2}$, where $\Phi_{0}=$ $2.07 \times 10^{-7} \mathrm{G} \mathrm{cm}^{2}$, the coherence length $\xi$ at the zero temperature is calculated as $2.38 \mathrm{~nm}$. It is well known that, for the two-gap superconductivity in the dirty limit, the impurity scattering could strongly enhance the upper critical field $H_{\mathrm{c} 2}$. This phenomenon has been clearly observed in $\mathrm{MgB}_{2}$, a dirty two-gap superconductor, where the $H_{\mathrm{c} 2}$ increases remarkably by alloying $\mathrm{MgB}_{2}$ with some impurities [33]. Therefore, the very high $H_{\mathrm{c} 2}$ value observed in our polycrystals could be reasonably attributed to the strong impurity scattering effect from the impurities in such a new mutiband superconductor.

Figure 3(a) shows magnetic hysteresis curves of this sample. A small amount of $\mathrm{Fe}_{7} \mathrm{Se}_{8}$ impurities may be the reason for the ferromagnetic background in $M-H$ curves. Shown in the inset is $M-H$ curve at $15 \mathrm{~K}$, which is higher than $T_{\mathrm{c}}$. Since the curves at $12.5 \mathrm{~K}$ (not shown) and $15 \mathrm{~K}$ are almost identical, we assumed this background is temperature-independent at low temperatures. In these hysteresis loops, the ratio between superconducting signals to the ferromagnetic background is not as strong as that of the sample prepared at higher temperatures [24]. This is because the magnetization is proportional to the grain sizes if the intragranular critical current density $\left(J_{\mathrm{c}}^{\text {intra }}\right)$ is fixed, and the grains prepared at higher temperatures are much larger. From the magnetization hysteresis loops, we can evaluate intragranular critical current density $J_{\mathrm{c}}^{\text {intra }}$ for polycrystalline samples using the Bean's model with the assumption of field-independent $J_{\mathrm{c}}$. According to Bean's model, $J_{\mathrm{c}}^{\text {intra }}\left[\mathrm{A} / \mathrm{cm}^{2}\right]$ is given by

$$
J_{\mathrm{c}}^{\text {intra }}=30 \frac{\Delta M}{d},
$$

with an assumption that intergranular critical current is zero, where $\Delta M[\mathrm{emu} / \mathrm{cc}]$ is $M_{\text {down }}-M_{\text {up }}$, $M_{\text {up }}$ and $M_{\text {down }}$ are the magnetization when sweeping field up and down, respectively, $d[\mathrm{~cm}]$ is the average diameter of the grain in the polycrystalline sample [34]. The $\Delta M$ used here is after subtracting the ferromagnetic background. Figure 3(b) shows the field dependences of the obtained $J_{\mathrm{c}}$ for this sample calculated from the data shown in figure 3(a) using Eq. (1) and the typical dimension. $J_{\mathrm{c}}$ calculated from $M-H$ curve is estimated to be $2 \times 10^{5} \mathrm{~A} / \mathrm{cm}^{2}$ at $5 \mathrm{~K}$ under zero field, this value is a little smaller than that in the single crystal [19], but still in the range for applications. $J_{\mathrm{c}}$ values in excess of $5 \times 10^{4} \mathrm{~A} / \mathrm{cm}^{2}$ are sustained up to $10 \mathrm{kOe}$.

Figures 4(b)-(e) depict MO images of the $\mathrm{FeTe}_{0.5} \mathrm{Se}_{0.5}$ polycrystalline sample in the remanent state after applying a 500 Oe field for 0.25 seconds which subsequently reduced down to zero. Figure 4(a) shows the polished surface of the $\mathrm{FeTe}_{1-\mathrm{x}} \mathrm{Se}_{\mathrm{x}}$ polycrystalline sample for MO measurements. The sample dimensions are $895 \times 555 \times 200 \mu \mathrm{m}^{3}$. Shown in figures 4 (b) to $4(\mathrm{e})$ are MO images of the remanent state recorded at several different temperatures. These images are similar with the MO images of 1111 polycrytals [35-37]. In these figures, the bright regions correspond to the trapped flux in the sample. The dots in these images are due to defects in the indicator garnet film. At all temperatures, the field profile is inhomogeneous, which implies that the intergranular current density is much smaller compared with the intragranular current density. The intragranular current density decreases gradually as the temperature is increased towards $T_{\mathrm{c}}$. We calculated the intragranular critical current density from the magnetic induction profile. Figure 4(f) shows the magnetic induction profiles along the dotted line in figure 4(b). In this calculation, we roughly estimate the intragranular critical 
current densities by $J_{\mathrm{c}} \sim \mathrm{d} B / \mathrm{d} x$. For typical grains, $J_{\mathrm{c}}$ thus estimated is $\sim 1 \times 10^{4} \mathrm{~A} / \mathrm{cm}^{2}$ at $5 \mathrm{~K}$. This value is much smaller than that estimated from the $M-H$ curve as shown in figure 3(b). This is because the formula we adopted here is based on the condition with infinite thickness of the sample, but from the SEM image, the ratio of width to thickness is very large. So the real $J_{\mathrm{c}}$ should be obtained by multiplying $w / t$, which is roughly 10 .

In figure 3 and figure 4, both $M-H$ and MO images of the remanent state for $\mathrm{FeTe}_{1-\mathrm{x}} \mathrm{Se}_{\mathrm{x}}$ polycrystalline sample only give the intragranular $J_{c}$. For practical applications and understanding the superconducting mechanism, global critical current density $J_{\mathrm{c}}$ should be investigated. In iron-based superconductors, global $J_{\mathrm{c}}$ for polycrystalline samples were less than $10^{4} \mathrm{~A} / \mathrm{cm}^{2}$, and very sensitive to magnetic field [38, 39]. Namely, weak-link feature is common in these systems. This has been usually ascribed to the tensile strain generated by dislocations located along the boundary [40] or competing orders, low carrier density and unconventional pairing symmetry [41]. We investigated the transport $J_{\mathrm{c}}$ of $\mathrm{FeTe}_{0.5} \mathrm{Se}_{0.5}$ polycrystalline sample. The sample dimension for this measurement is $1200 \times 180 \times 75 \mu^{3}$, and the distance between two voltage contacts is $470 \mu \mathrm{m}$. In superconductors, driving force competes with pinning force. If the former wins over the latter, measurable voltages could be observed. Inset of figure 5(a) shows the zero-field $I-V$ characteristics at different temperatures ranging from $4.2 \mathrm{~K}$ to $10 \mathrm{~K}$. Here we adopt $E=1 \mu \mathrm{V} / \mathrm{cm}$ as a criterion for the $I$ $-V$ curve to define transport $J_{\mathrm{c}}$. Figure 5(a) shows the temperature dependence of transport $J_{\mathrm{c}}$ at zero field. At $4.2 \mathrm{~K}$, a transport $J_{\mathrm{c}}$ as high as $724 \mathrm{~A} / \mathrm{cm}^{2}$ was observed in the $\mathrm{FeTe}_{1-\mathrm{x}} \mathrm{Se}_{\mathrm{x}}$ polycrystalline sample. Transport $J_{\mathrm{c}}$ does not increase so fast below $T_{\mathrm{c}}$. Transport $J_{\mathrm{c}}$ as a function of field at $4.2 \mathrm{~K}$ is shown in figure 5(b). Similar to $\mathrm{YBa}_{2} \mathrm{Cu}_{3} \mathrm{O}_{7-\mathrm{y}}$ and other iron-based superconductors, transport $J_{\mathrm{c}}$ shows strong field dependence at low fields. For example, $J_{\mathrm{c}}(0$ Oe) is 10 times as large as $J_{\mathrm{c}}(0.5 \mathrm{kOe})$. When the field is more than $0.5 \mathrm{kOe}$, the field dependence of $J_{\mathrm{c}}$ is not as strong as that in the low field region. The value of transport $J_{\mathrm{c}}$ is more than two orders less than the intragranular $J_{\mathrm{c}}$ as shown in the figure 3(b) and that obtained from the magnetic induction profile in figure 4(e). This implies the presence of weak links between superconducting grains. The transport $J_{\mathrm{c}}$ of $\mathrm{FeTe}_{1-\mathrm{x}} \mathrm{Se}_{\mathrm{x}}$ polycrystalline sample is smaller than $\mathrm{SmFeAsO}_{0.7} \mathrm{~F}_{0.3-\delta}$ wires $\left(1300 \mathrm{~A} / \mathrm{cm}^{2}\right)$ [38] and $\mathrm{Ag}$ doped $\mathrm{Sr}_{0.6} \mathrm{~K}_{0.4} \mathrm{Fe}_{2} \mathrm{As}_{2}$ wires $\left(3750 \mathrm{~A} / \mathrm{cm}^{2}\right)$ [39], but the same order as randomly oriented polycrytalline $\mathrm{YBa}_{2} \mathrm{Cu}_{3} \mathrm{O}_{7-\mathrm{y}}$ [42], but several times larger than that in $\mathrm{FeTe}_{0.5} \mathrm{Se}_{0.5}$ wires [21-23].

Figures 6(a) to 6(d) reveal the penetration of vortices at $5 \mathrm{~K}$ in the $\mathrm{FeTe}_{0.5} \mathrm{Se}_{0.5}$ polycrystalline sample. In figure 6(a), at low field of 2 Oe, most part of the sample was still in the Meissner state. When the field was increased to 10 Oe, the flux profile shows a pattern similar to the critical state as shown in figure 6(c). When the field was further increased to 20 Oe, the sample was fully penetrated. For samples prepared at higher temperature [21], when the field was as low as $1 \mathrm{Oe}$, magnetic flux penetrates intergranular regions. The shapes of the superconducting grains are more visible when the field is increased. This also indicated the weak-link feature in this lower temperature prepared sample is much reduced compared with the higher temperature prepared sample [21]. The intergranular $J_{\mathrm{c}}$ for a thin superconductor can be roughly estimated by $J_{\mathrm{c}} \sim \Delta B / t$, where $\Delta B$ is the difference in local induction between the centre and edge of the sample and $t$ is the thickness of the sample. With $\Delta B \sim 10 \mathrm{G}$ and $t=$ $200 \mu \mathrm{m}, J_{\mathrm{c}}$ is estimated to be $500 \mathrm{~A} / \mathrm{cm}^{2}$ at $5 \mathrm{~K}$, which is consistent with the value $440 \mathrm{~A} / \mathrm{cm}^{2}$ measured from transport measurement (figure 5(a)).

In superconducting wires, polycrystalline samples with smaller grain size are more promising. Our results show that both grain size and weak-link feature has been reduced in the polycrystalline $\mathrm{FeTe}_{0.5} \mathrm{Se}_{0.5}$ samples prepared at $550^{\circ} \mathrm{C}$ compared with the samples prepared at higher temperatures. By using higher static pressure to make denser pellets and increasing the annealing time, or using high-pressure synthesis method we can obtain more pure and homogenous samples with higher transport critical current density. Alternatively, we can utilize the powder-in-tube method to make the sample more dense and well-connected. This is the subject of ongoing research. 
In conclusion, X-ray diffraction, magnetization, resistivity, transport critical current density and magneto-optical measurements were performed on high quality polycrystalline $\mathrm{FeTe}_{0.5} \mathrm{Se}_{0.5}$ prepared at $550^{\circ} \mathrm{C}$. The transport critical current density over $700 \mathrm{~A} / \mathrm{cm}^{2}$ at $4.2 \mathrm{~K}$ under zero field is obtained. This value is several times larger than that of $\mathrm{FeTe}_{0.5} \mathrm{Se}_{0.5}$ superconducting wires, although it is more than two orders less than the intragranular critical current density. $J_{\mathrm{c}}$ estimated from magneto-optical (MO) images of flux penetration is also similar to this value. The upper critical field $H_{\mathrm{c} 2}$ of the polycrystalline $\mathrm{FeTe}_{0.5} \mathrm{Se}_{0.5}$ is $585 \mathrm{kOe}$, which is comparable to that of single crystals. Our low-temperature synthesis is promising to the development of practical $\mathrm{FeTe}_{1-\mathrm{x}} \mathrm{Se}_{\mathrm{x}}$ system, with potential higher transport critical current density, for applications especially for superconducting wires.

\section{References}

[1] Kamihara Y, Watanabe T, Hirano M and Hosono H 2008 J. Am. Chem. Soc. 1303296

[2] Chen G F, Li Z, Wu D, Dong J, Li G, Hu W Z, Zheng P, Luo J L and Wang NL 2008 Phys. Rev. Lett. 100247002

[3] Cheng P, Fang L, Yang H, Zhu X Y, Mu G, Luo H Q, Wang Z S and Wen H H 2008 Sci. China Ser. G 51719

[4] Ren Z A, Che G C, Dong X L, Yang J, Lu W, Yi W, Shen X L, Li Z C, Sun L L, Zhou F and Zhao Z X Europhys. Lett. 20088317002

[5] Rotter M, Tegel M and Johrendt D 2008 Phys. Rev. Lett. 101107006

[6] Wang X C, Liu Q Q, Lv Y X, Gao W B, Yang L X, Yu R C, Li F Y and Jin C Q 2008 Solid State Commun. 148538

[7] Hsu F C, Luo J Y, Yeh K W, Chen T K, Huang T W, Wu P M, Lee Y C, Huang Y L, Chu Y Y, Yan D C and Wu M K 2008 Proc. Natl. Acad. Sci. 10514262

[8] Ogino H, Katsura Y, Horii S, Kishio K and Shimoyama J 2009 Supercond. Sci. Technol. 22085001

[9] Medvedev S, McQueen T M, Troyan I A, Palasyuk T, Eremets M I, Cava R J, Naghavi1 S, Casper1 F, Ksenofontov1 V, Wortmann G and Felser C 2009 Nat. Mater. 8630

[10] Millican J N, Phelan D, Thomas E L, Leão J B and Carpenter E 2009 Solid State Commun. 149707

[11] Guo J G, Jin S F, Wang G, Wang S C, Zhu K X, Zhou T T, He M and Chen X L 2010 Phys. Rev. B 82 10520(R)

[12] Krzton-Maziopa A, Shermadini Z, Pomjakushina E, Pomjakushin V, Bendele M, Amato A, Khasanov R, Luetkens H, Conder K 2010 arXiv:1012.3637

[13] Wang A F, Ying J J, Yan Y J, Liu R H, Luo X G, Li Z Y, Wang X F, Zhang M, Ye G J, Cheng P, Xiang Z J and Chen X H 2010 arXiv:1012.5525

[14] Yeh K W, Ke C T, Huang T W, Chen T K, Huang Y L, Wu P M and Wu M K 2009 Crystal Grwoth \& Design. 94847

[15] Yadav C S and Paulose P L 2009 New J. Phys. 11103046

[16] Kida T, Matsunaga T, Hagiwara M, Mizuguchi Y, Takano Y and Kindo K 2009 J. Phys. Soc. Jpn. 78113701

[17] Braithwate D, Lapertot G, Knafo W and Sheikin I 2010 J. Phys. Soc. Jpn. 79053703

[18] Taen T, Nakajima Y and Tamegai T 2010 Physica C 470 S391

[19] Taen T, Tsuchiya Y, Nakajima Y and Tamegai T 2009 Phys. Rev. B 80092502

[20] Taen T, Nakajima Y and Tamegai T 2010 Physica C 4701106

[21] Mizuguchi Y, Deguchi K, Tsuda S, Yamaguchi T, Takeya H, Kumakura H and Takano Y 2009 Appl. Phys. Express 2083004

[22] Ozaki T, Deguchi K, Mizuguchi Y, Kumakura H and Takano Y 2010 arXiv:1008.1447

[23] Ozaki T, Deguchi K, Mizuguchi Y, Kawasaki Y, Tanaka T, Yamaguchi T, Tsuda S, Kumakura H and Takano Y 2011 arXiv:1103.0402

[24] Ding Q, Taen T, Mohan S, Nakajima Y and Tamegai T 2011 Physica C accepted

[25] Soibel A, Zeldov E, Rappaport M, Myasoedov Y, Tamegai T, Ooi O, Konczykowski M and 
Geshkenbein V B 2000 Nature 406282

[26] Yasugaki M, Itaka K, Tokunaga M, Kameda N, Tamegai T 2002 Phys. Rev. B 65212502

[27] Sales B C, Sefat A S, McGuire M A, Jin R Y and Mandrus D 2009 Phys. Rev. B 79 094521

[28] Yeh K W, Huang T W, Huang Y L, Chen T K, Hsu F C, Wu P M, Lee Y C, Chu Y Y, Chen C L, Luo J Y, Yan D C and Wu M K 208 Europhys. Lett. 8437002

[29] Mizuguchi Y, Tomioka F, Tsuda S, Yamaguchi T and Takano Y 2009 J. Phys. Soc. Jpn. 78074712

[30] Awana S, Pal A, Vajpayee A, Mudgel M, Kishan H, Husain M, Zeng R, Yu S, Guo Y F, Shi Y G, Yamaura K, Takayama-Muromachi E 2010 J. Appl. Phys. 107 09E128

[31'] Liu T J, Ke X, Qian B, Hu J, Fobes D, Vehstedt E K, Pham H, Yang J H, Fang M H, Spinu L, Schiffer P, Liu Y and Mao Z Q 2009 Phys. Rev. B, 80174509

[32] Werthamer N R, Helfand E and Hohenberg P C 1966 Phys. Rev. 147295

[33] Gurevich A, Patnaik S, Braccini V, Kim K H, Mielke C, Song X, Cooley L D, Bu S D, Kim D M, Choi J H,Belenky L J, Giencke J, Lee M K, Tian W, Pan X Q, Siri A, Hellstrom E E, Eom C B and. Larbalestier D C 2004 Supercond. Sci. Technol. 17278

[34] Bean C P 1964 Rev. Mod. Phys. 3631

[35] Tamegai T, Nakajima Y, Tsuchiya Y, Iyo A, Miyazawa K, Shirage M P, Kito H and Eisaki H 2008 J. Phys. Soc. Jpn. Suppl. C 7754

[36] Tamegai T, Nakajima Y, Tsuchiya Y, Iyo A, Miyazawa K, Shirage M P, Kito H and Eisaki H 2008 Physicsa C 469915

[37] Nakajima Y, Maruoka T, Tamegai T, Kamihara Y, Hirano M and Hosono H Physica C 470 S406

[38] Wang L, Qi Y P, Wang D L, Gao Z S, Zhang X P, Zhang Z Y, Wang C L and Ma Y W 2010 Supercond. Sci. Technol. 23075005

[39] Qi Y P, Wang L, Wang D L, Zhang Z Y, Gao Z S, Zhang X P and Ma Y 2010 Supercond. Sci. Technol. 23055009

[40] Deutscher G 2010 Appl. Phys. Lett. 96122502

[41] Lee S, Jiang J, Weiss J D, Folkman C M, Bark C W, Tarantini T, Xu A, Abraimov D, Polyanskii A, Nelson C T, Zhang Y, Baek S H, Jang H W, Yamamoto A, Kametani F, Pan X Q, Hellstrom E E, Gurevich A, Eom C B and Larbalestier D C 2009 Appl. Phys. Lett. 95212505

[42] Li S, Fistl M, Deak J, Mtecalf P and McElfresh M 1995 Phys. Rev. B 52 R747 

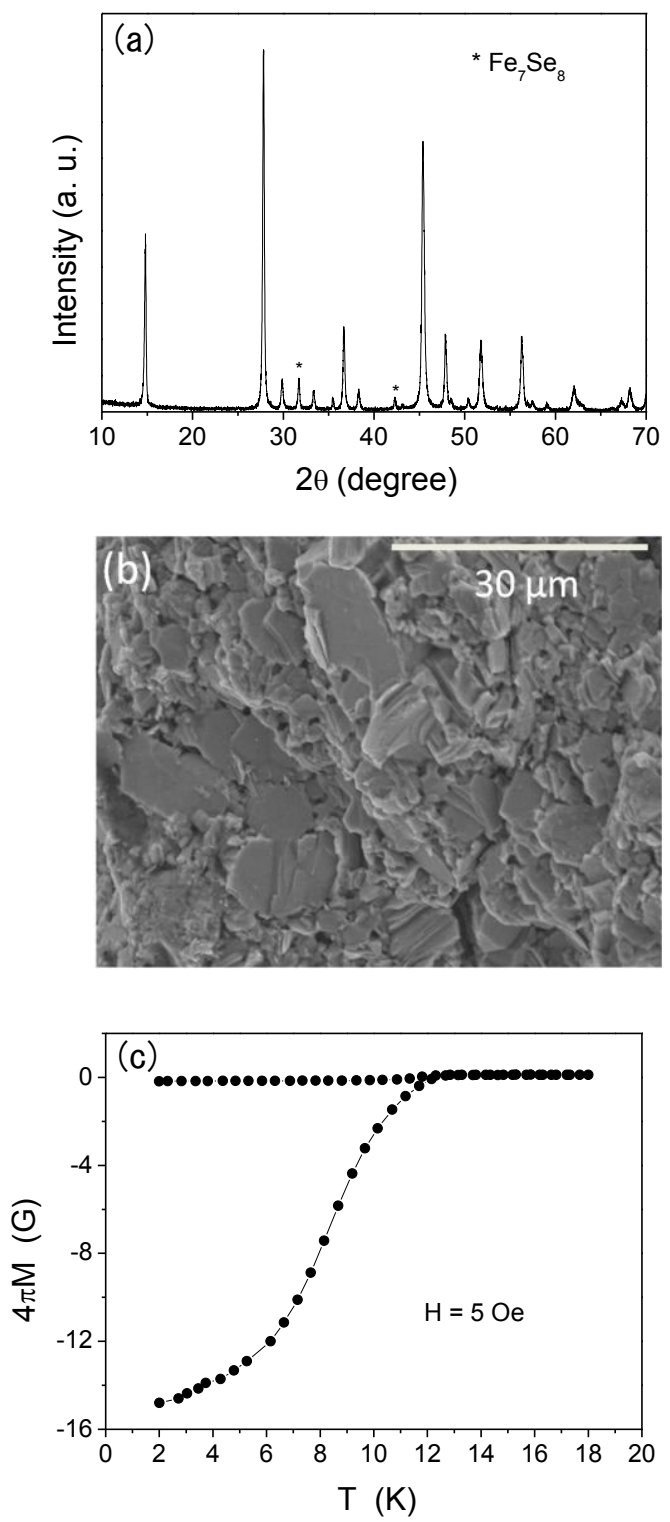

Figure1. (a) Powder X-ray diffraction pattern of as-prepared $\mathrm{FeTe}_{0.5} \mathrm{Se}_{0.5}$ polycrystalline sample. (b) SEM image of the $\mathrm{FeTe}_{0.5} \mathrm{Se}_{0.5}$ polycrystalline sample. (c) Temperature dependence of magnetic susceptibility of $\mathrm{FeTe}_{0.5} \mathrm{Se}_{0.5}$ polycrystalline sample measured at 5 Oe. 

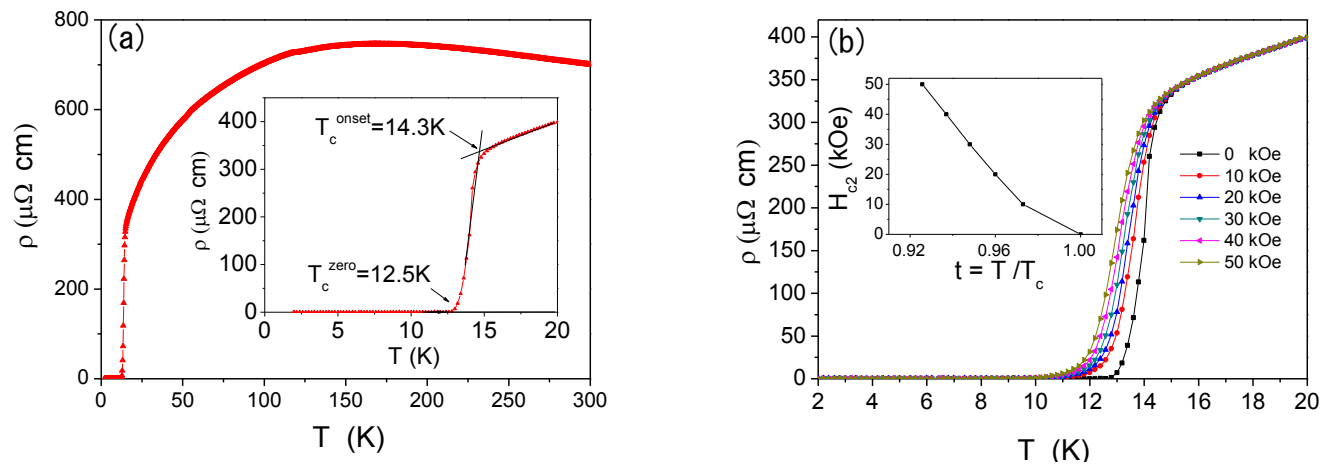

Figure 2. (a) Temperature dependence of the resistivity of $\mathrm{FeTe}_{0.5} \mathrm{Se}_{0.5}$ polycrystalline sample. Inset shows the resistivity around $T_{\mathrm{c}}$. (b) Field dependence of the resistivity of $\mathrm{FeTe}_{0.5} \mathrm{Se}_{0.5}$ polycrystalline sample around $T_{\mathrm{c}}$. Inset shows the upper critical field $H_{\mathrm{c} 2}$ versus temperature determined by the midpoint of the resistive transition. 

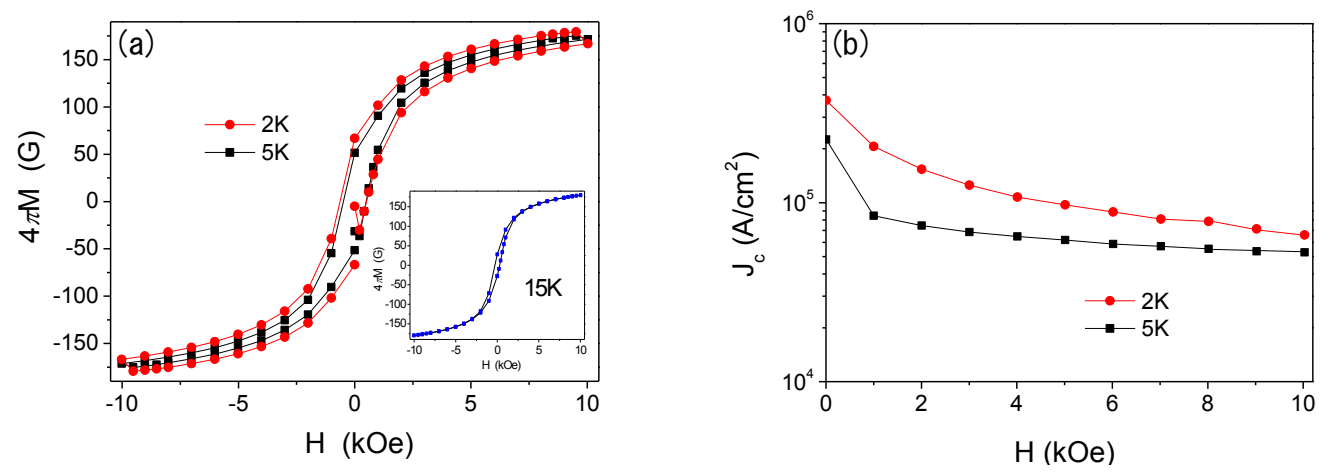

Figure 3. (a) Magnetic field dependence of magnetization at different temperatures for $\mathrm{FeTe}_{0.5} \mathrm{Se}_{0.5}$ polycrystalline sample. Inset shows $M-H$ curves at $15 \mathrm{~K}$. (b) Magnetic field dependence of critical current densities calculated from the data in (a). 

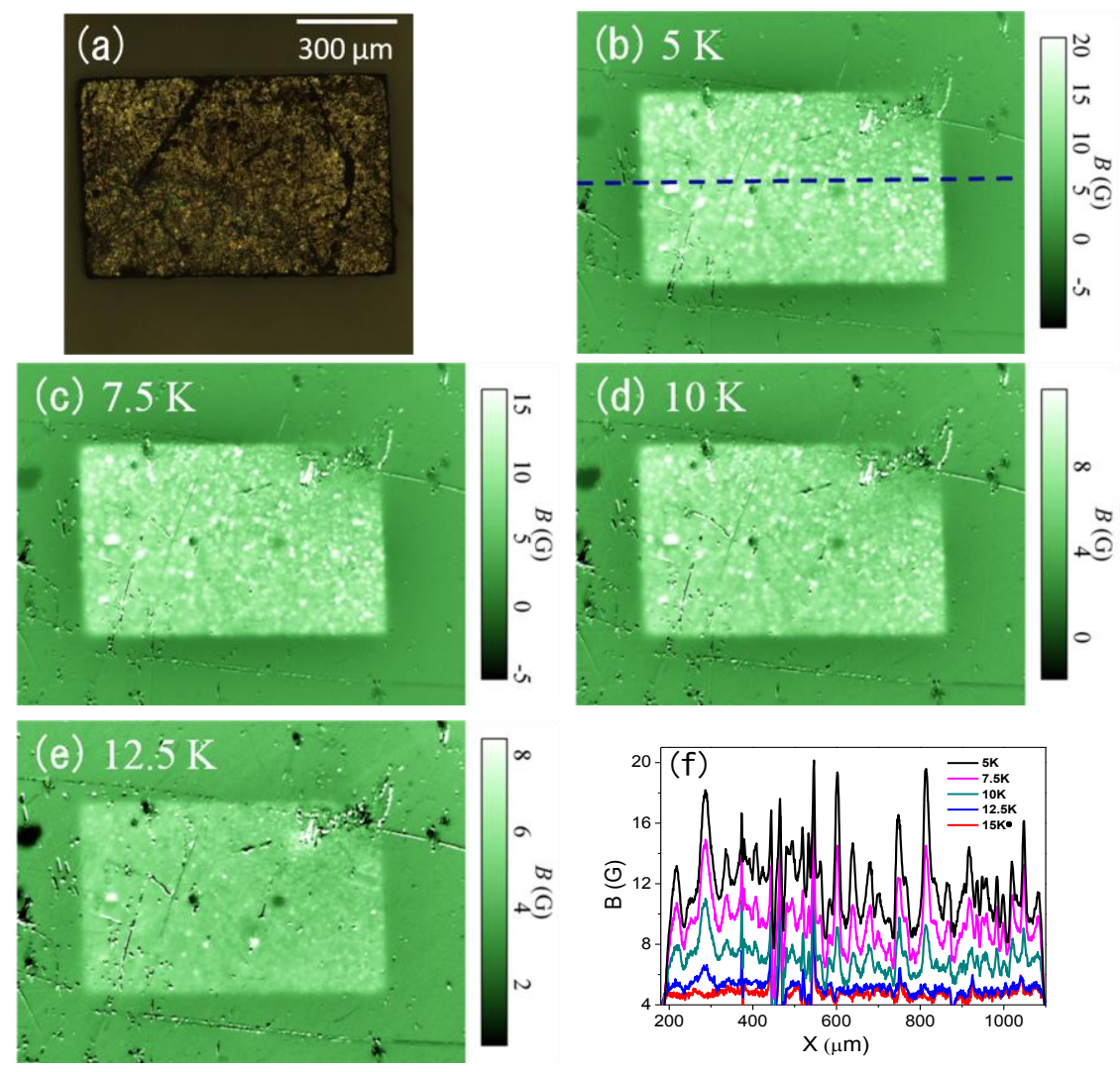

Figure 4. (a) Optical images of the $\mathrm{FeTe}_{0.5} \mathrm{Se}_{0.5}$ polycrystalline sample used for $\mathrm{MO}$ imaging. Differential MO images in the remanent state of the $\mathrm{FeTe}_{0.5} \mathrm{Se}_{0.5}$ polycrystalline sample at (b) $5 \mathrm{~K}$, (c) $7.5 \mathrm{~K}$, (d) $10 \mathrm{~K}$, and (e) $12.5 \mathrm{~K}$ after cycling the field up to $500 \mathrm{Oe}$ for 0.25 seconds, (f) the local magnetic induction profiles at different temperatures taken along the dotted lines in (b). 

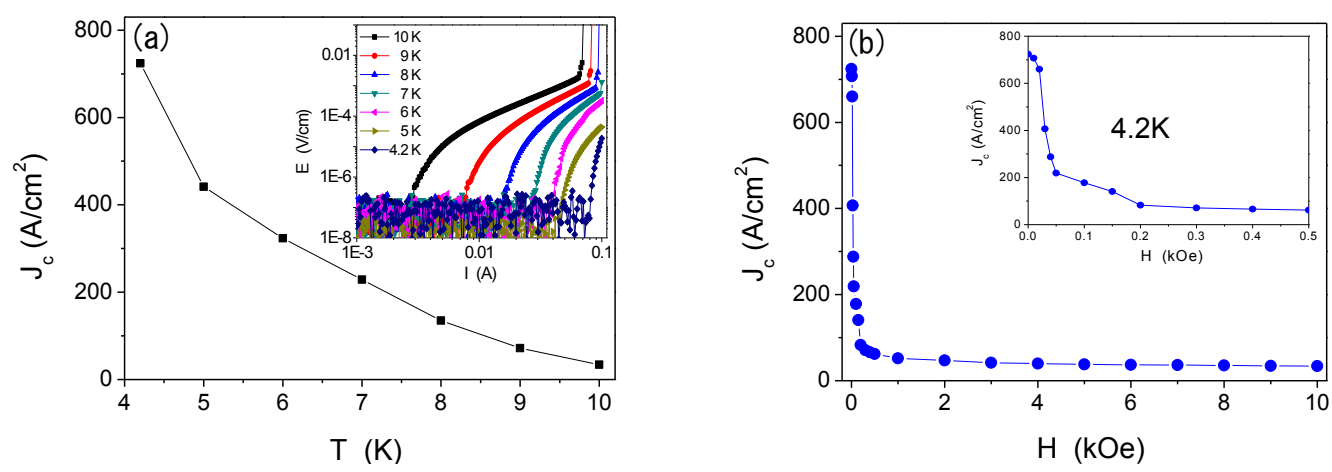

Figure 5. (a) Temperature dependence of the transport critical current density $J_{c}$ in $\mathrm{FeTe}_{0.5} \mathrm{Se}_{0.5}$ polycrystalline sample at zero field. Inset shows $I-V$ characteristics in $\mathrm{FeTe}_{0.5} \mathrm{Se}_{0.5}$ polycrystalline sampl at $T=4.2,5,6,7,8,9$ and $10 \mathrm{~K}$. (b) Field dependence of the transport $J_{\mathrm{c}}$ in $\mathrm{FeTe}_{0.5} \mathrm{Se}_{0.5}$ polycrystalline sample at $4.2 \mathrm{~K}$. Inset shows $J_{\mathrm{c}}$ at lower fields. 

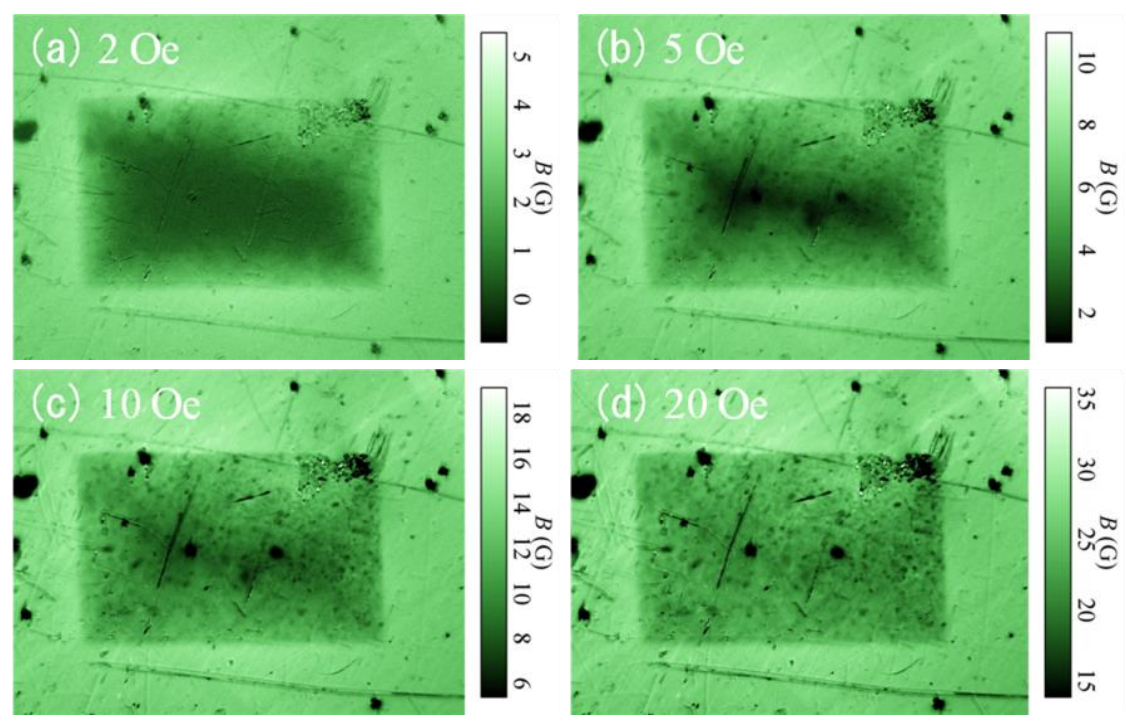

Figure 6. MO images of flux penetration into $\mathrm{FeTe}_{0.5} \mathrm{Se}_{0.5}$ polycrystalline sample at (a) $2 \mathrm{Oe}$, (b) $5 \mathrm{Oe}$, (c) $10 \mathrm{Oe}$, (d) 20 Oe after zero-field cooling down to $5 \mathrm{~K}$. 